Received: 10 May 2017

Accepted: 31 July 2017

Published online: 25 August 2017

CIENTIFIC REP

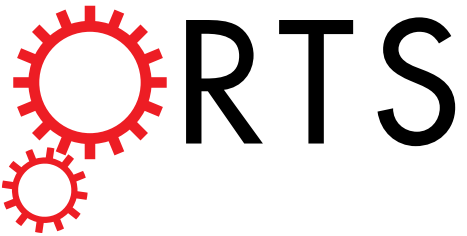

\title{
OPEN Routes to probe Bismuth induced strong-coupling superconductivity in bimetallic Biln alloys
}

Ashish Chhaganlal Gandhi \& ShengYun Wu (iD

We report the observation of strong electron-phonon coupling in intergranular linked Biln superconductors over an infinite range mediated by low-lying phonons. An enhanced superconducting transition temperature was observed from the magnetization, revealing a main diamagnetic Meissner state below $\mathrm{T}_{\mathrm{C}}(0)=5.86(1) \mathrm{K}$ and a critical field $\mathrm{H}_{\mathrm{C}}(0)=1355(15)$ Oe with an $\ln _{2}$ Bi phase of the composite sample. The electron-phonon coupling to low lying phonons is found to be the leading mechanism for observed strong-coupling superconductivity in the Biln system. Our findings suggest that $\ln _{2} \mathrm{Bi}$ is in the strong-coupling region with $\mathrm{T}_{\mathrm{C}}(0)=5.62(1) \mathrm{K}, \lambda_{\mathrm{ep}}=1.45, \omega_{\mathrm{ln}}=45.92 \mathrm{~K}$ and $\alpha=2.23$. The estimated upper critical field can be well-described by a power law with $\alpha$ value higher than 2 , consistent with the strong electron-phonon coupling.

Recently a number of electron-phonon coupled bi- and tri-metallic superconductors with enhanced superconducting transition have been discovered, renewing interest in conventional phonon-mediated superconductivity ${ }^{1-4}$. We consider the BiIn bimetallic system, in which $\mathrm{Bi}$ is a semimetal and In is a weak-coupled superconductor with transition temperature $\mathrm{T}_{\mathrm{C}}(0)=3.4 \mathrm{~K}$ (at ambient atmosphere). When annealed together they form $\operatorname{In}_{2} \mathrm{Bi} \mathrm{In}_{5} \mathrm{Bi}_{3}$ and $\operatorname{InBi}$ bimetallic compounds and a solid solution, $\alpha$ - $\operatorname{In}^{5}$. The reported $\mathrm{T}_{\mathrm{C}}(0)$ for single crystals of $\operatorname{In}_{2} \mathrm{Bi}$ and $\mathrm{In}_{5} \mathrm{Bi}_{3}$ are $5.1 \mathrm{~K}$ and $4.1 \mathrm{~K}$, respectively, whereas $\mathrm{InBi}$ is a non-superconductor down to $0.5 \mathrm{~K}$. Hutcherson et al. ${ }^{6}$ reported the two-gap superconductivity in a composite $\left(\operatorname{In}_{2} \mathrm{Bi}+\operatorname{In}_{5} \mathrm{Bi}_{3}\right)$ superconductor with $\mathrm{T}_{\mathrm{Cl}}(0)=5.6 \mathrm{~K}$ and $\mathrm{T}_{\mathrm{C} 2}(0)=4.1 \mathrm{~K}$. A microscopic examination of polished InBi samples revealed a heterogeneous mixture of crystal grains, except for pure crystalline phases indicating granular superconductivity. In such a system, the Josephson tunneling between superconducting grains establishes inter-granular links and results in macroscopic superconductivity ${ }^{7}$. However, a comprehensive study, including electron-phonon coupling strength and the enhanced $\mathrm{T}_{\mathrm{C}}$ in the InBi system, has not yet been carried out. Conventionally, BCS-Eliashberg theory showed that $T_{C}$ is the combined effect of electron-phonon coupling strength $\left(\lambda_{e p}\right)$ and phonon energy $\left(\omega_{l n}\right)$. In this study, we present a transition of weak to strong electron-coupling superconductivity by tuning the Bi concentration in bimetallic $\mathrm{Bi}_{\mathrm{y}} \mathrm{In}_{1-\mathrm{y}}$ alloys, consisting of two superconducting gaps at $\mathrm{y}=0.1,0.2,0.6$, and 0.7 and a single gap at $\mathrm{y}=0.01$, $0.2,0.3,0.4$ and 0.5 , respectively. The enhancement of $\mathrm{T}_{\mathrm{C}}(0)=5.62(1) \mathrm{K}$ was observed at $\mathrm{y}=0.3$, led by low lying phonons that can be well described by using Allen and Dynes' theory ${ }^{2}$.

\section{Results}

Crystal structure analysis of Biln alloy. Figure 1(a) presents the typical EDS spectra, showing a series of elemental $\mathrm{Bi}$ and In constituents that can be assigned to $\mathrm{Bi}-\mathrm{Ma}_{1}, \mathrm{In}-\mathrm{L} \alpha_{1}, \mathrm{In}-\mathrm{L} \beta_{1}$, and In- $\mathrm{L} \beta_{2}$, respectively. The small peaks of $\mathrm{C}$ and $\mathrm{Cu}$ were the result of the carbon film on the $\mathrm{Cu}$ grid from mounting the sample. Moreover, the atomic percentage of $\mathrm{Bi}$ increases linearly with a slope of 106(3) $\mathrm{Bi}$ at. \%/y and approaches the value of bulk $\mathrm{Bi}(\mathrm{y}>0.9)$, as shown in Fig. 1(b). The observed atomic \% Bi is in good agreement with initial composition y. The structural analysis was conducted by room temperature X-ray diffraction (XRD) using synchrotron radiation (BL-01C2) at the National Synchrotron Radiation Research Center in Taiwan, with an incident wavelength of $\lambda=0.7749 \AA$. For detailed investigation of the crystalline phase of bimetallic alloys, the SR-XRD technique must be employed, as this is otherwise quite difficult using the usual XRD techniques. A 2D plot of the XRD pattern of $\mathrm{Bi}_{\mathrm{y}} \operatorname{In}_{1-\mathrm{y}}(0.01 \leq \mathrm{y} \leq 0.99)$ bimetallic alloys over a narrow scattering range of $2 \theta$ is as shown in Fig. $1(\mathrm{c})$, where the vertical axis represents the starting composition y. Different colors were used to differentiate the peak intensities of the diffraction pattern. From the above $2 \mathrm{D}$ plot, for $\mathrm{y}=0.01$ a single nuclear peak (101) indexed based on I4/ $\mathrm{mmm}$ (No. 139) becomes visible, indicating the formation of an In-solid solution having the same structure as 

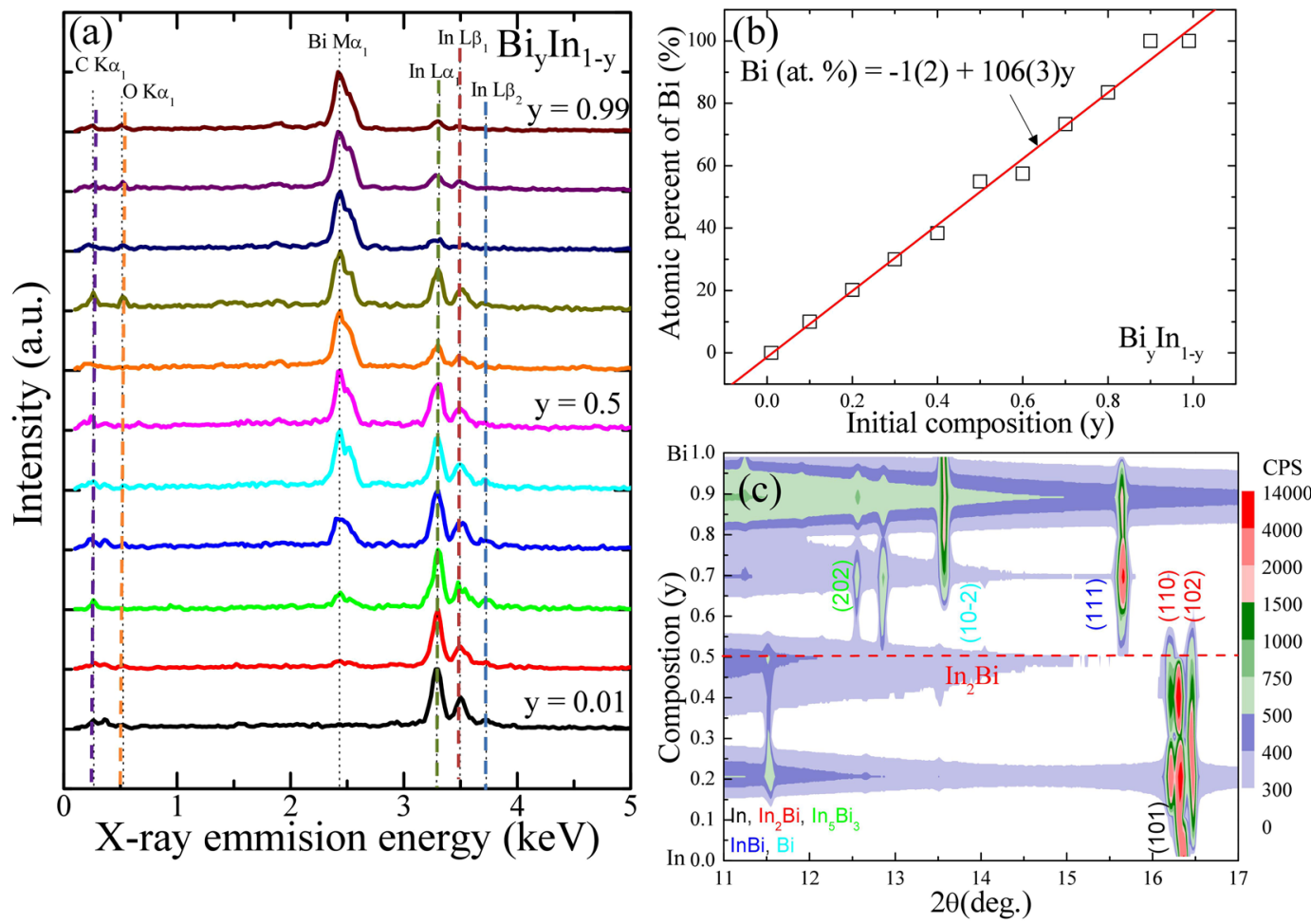

Figure 1. The EDS and x-ray analysis. (a) Typical EDS elemental spectra, revealing a series of peaks associated with elemental Bi and In, verifying that all samples contain only Bi and In elements. (b) Plot of bismuth atomic percentage verses initial composition $y$, where the solid line is a linear fit to the data points. (c) A 2D plot of XRD patterns of $B \mathrm{Bi}_{\mathrm{y}} \mathrm{In}_{1-\mathrm{y}}$ bimetallic alloys over a narrow $2 \theta$ scattering range.

that of virgin In. Since the atomic radii of $\mathrm{Bi}(156 \mathrm{pm})$ is smaller than In (167 pm), Bi can therefore occupy interstitial sites in the In lattice and can form an interstitial solid solution ${ }^{8}$. The In phase becomes unstable beyond $y=0.1$ and shows the presence of two immiscible phases in each alloy up to $y=0.4$. A horizontal red-colored dashed line drawn at $y=0.5$ marks the two nuclear peaks (110) and (102) indexed based on $P 6_{3} / m m c$ (No. 194), indicating the formation of a pure $\operatorname{In}_{2} B i$ crystalline phase. For $\mathrm{y}=0.6$, two additional nuclear peaks (202) and (111) become visible indexed based on $I 4 / \mathrm{mcm}$ (No. 140) and P4/ $\mathrm{nmm}$ (No. 129), respectively indicating the formation of immiscible $\mathrm{In}_{5} \mathrm{Bi}_{3}$ and InBi crystalline phases. For y from 0.7 to 0.99 , along with InBi one additional nuclear peak (10-2) becomes visible indexed based on $R-3 m$ (No. 166), and trigonal-hexagonal scalenohedral Bi indicates the presence of two immiscible phases in each alloy. In bimetallic alloys, because of the different atomic and structural properties of the constituent's elements and the state of the alloy (pure or mixed), lattice expansion or contraction is unavoidable. The detailed structural analysis of the $\mathrm{x}$-ray diffraction pattern of $\mathrm{Bi}_{\mathrm{y}} \mathrm{In}_{1-\mathrm{y}}$ bimetallic alloys was further refined by Rietveld analysis ${ }^{9}$ using the GSAS software package ${ }^{10}$. The refined patterns and the fitting parameters are as shown in Fig. 2. From the refined XRD spectra of $\mathrm{Bi}_{\mathrm{y}} \mathrm{In}_{1-\mathrm{y}}$ bimetallic alloys, it was observed that depending on their initial composition, formed alloys are either in pure or mixed form. A single phase was obtained at $y=0.01$, as shown in Fig. 2(a), exhibiting the expected In (S) solid solution structure. Since the atomic radii of $\mathrm{Bi}$ is smaller than In, Bi can therefore occupy interstitial sites in the In lattice and form an interstitial solid solution. The major detectable phase was $\operatorname{In}(\mathrm{S})$, along with $\operatorname{In}_{2} \mathrm{Bi}(\mathrm{S})$ as a secondary phase, for the region of $\mathrm{y}=0.1$ to 0.4 , presenting two immiscible phases in this concentration region, as shown in Fig. 2(b-e). After this stage, the reactant phases have converted fully into $\operatorname{In}_{2} \mathrm{Bi}(\mathrm{S})$ for $\mathrm{x}=0.5$, as shown in Fig. 2 (f). It is to be noted that there is a retardation in the growth of $\operatorname{In}_{2} \mathrm{Bi}(\mathrm{S})$ as the percentage of $\mathrm{Bi}$ increases. Structural transformation was observed as $y$ is larger than 0.5 , and then a new immiscible phase including $\operatorname{In}_{5} \mathrm{Bi}_{3}(\mathrm{~S})$ and $\mathrm{InBi}(\mathrm{N})$ was detected at $y=0.6$, forming a co-existence of a superconductor $(S)$ and a normal $(\mathrm{N})$ bimetallic compound, and the pairing correlation between electrons is delivered from the superconducting phase to the normal phase through proximity effect (PE), as shown in Fig. 2(g). Figure S1(a) represents the observed state of the alloy (i.e. pure or mixed phases) with respect to initial composition $y$ and the corresponding crystal structure of $\operatorname{In}, \operatorname{In}_{2} \mathrm{Bi}$, $\mathrm{In}_{5} \mathrm{Bi}_{3}$, InBi and $\mathrm{Bi}$, respectively (see the supporting information: Figure $\mathrm{S} 1$ ). The formed pure $\operatorname{In}_{2} \mathrm{Bi}$ phase at $\mathrm{y}=0.5$, is marked by a red circle. The observed newly formed compounds with respect to the initial composition contradict with previous finding. Giessen et al. ${ }^{11}$ reported the equilibrium phase diagram of In-Bi according to which pure $\operatorname{In}_{2} \mathrm{Bi}, \mathrm{In}_{5} \mathrm{Bi}_{3}$ and $\mathrm{InBi}$ are formed at 33.5(0.5), 37.5(5) and 50 atomic $\%$ of $\mathrm{Bi}$. The reason for the observed discrepancy is not clear yet, but for our alloys the estimated atomic \% and structural properties are in good agreement with the initial In-Bi composition. The effect of Bi doping in In (solid solution) and the effect of strain on the lattice constants of the In-solid solution, $\operatorname{In}_{2} \mathrm{Bi}, \operatorname{In}_{5} \mathrm{Bi}_{3}$ and $\mathrm{InBi}$, is plotted with respect to initial composition y, as shown in Figure S1(b-e) (the dotted line represents the standard values ${ }^{5}$ ). Lattice expansion was 

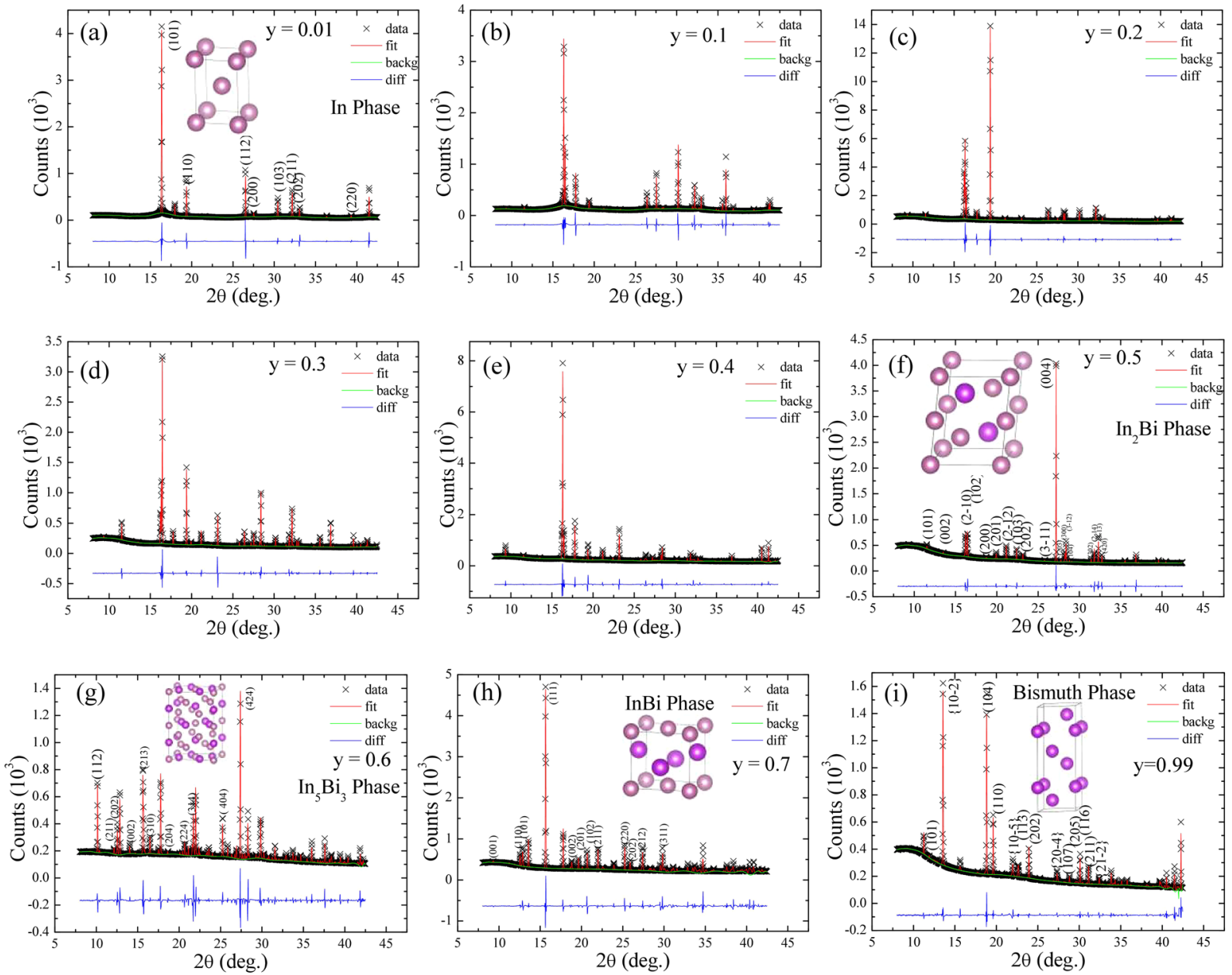

Figure 2. The crystal structure and $\mathrm{x}$-ray refinements analysis. (a-g) The observed and Rietveld refined (gray solid line) X-ray diffraction patterns of $\mathrm{Bi}_{\mathrm{y}} \mathrm{In}_{1-\mathrm{y}}$ bimetallic alloys.

observed for the In-solid solution, InBi and $\mathrm{Bi}$ phases, and contraction was observed for the $\operatorname{In}_{5} \mathrm{Bi}_{3}$ phase of the mixed phase alloys. For $\mathrm{In}_{2} \mathrm{Bi}$, phase lattice contraction was observed along the axial plane, and expansion along the basal plane. The observed lattice expansion for the solid solution is the combined effects of electron doping, as $\mathrm{Bi}$ has one extra electron compared to In and the strain. On the other hand, the observed lattice expansion/ contraction in immiscible mixed alloys could be due to strain effect, since thermal coefficients vary from material to material. The atomic position and structural properties of the parent elements, newly formed $\operatorname{In}_{2} \operatorname{Bi}(y=0.5)$, $\mathrm{In}_{5} \mathrm{Bi}_{3}(\mathrm{y}=0.6)$, and $\mathrm{InBi}(\mathrm{y}=0.6)$ crystalline phases obtained after refinement are as tabulated in the supplementary information of Table S1, along with the atomic radii of In and Bi. The observed lattice constants of the different crystalline phases are in excellent agreement with previously published results. As tabulated in Table S1, it can be observed that the $c / a$ of $\operatorname{In}_{2} \mathrm{Bi}, \operatorname{In}_{5} \mathrm{Bi}_{3}$ and $\mathrm{InBi}, 1.1992,1.4830$ and 0.9548 , respectively, is smaller than that of both In and $\mathrm{Bi}, 1.5208$ and 2.6025, respectively. Such a contracted lattice, compared to that of the constituent elements, may result in softening of the vibrational mode, that is, phonon softening, thus giving rise to the enhanced electron-phonon coupling strength. However, it has been reported that $\operatorname{In}_{2} \mathrm{Bi}$ and $\operatorname{In}_{5} \mathrm{Bi}_{3}$ are superconductors below 5.1 and $4.1 \mathrm{~K}$, respectively, whereas $\mathrm{InBi}$ is non-superconducting down to $0.5 \mathrm{~K}^{6}$. Allen et al. ${ }^{2}$ observed strong-coupling behavior for the $\operatorname{In}_{2}$ Bi phase mediated by low energy phonons. Therefore, studying the superconducting properties of In-Bi bimetallic alloys will provide more insight into these newly formed compounds.

Temperature Dependency of ZFC and FC Magnetization. A superconducting material when cooled below unique superconducting transition temperature $\mathrm{T}_{\mathrm{C}}$ shows perfect Meissner effect. However in $\mathrm{Bi}_{\mathrm{y}} \mathrm{In}_{1-\mathrm{y}}$ bimetallic alloys, the effect of electron doping, strain effect, and superconducting proximity (SPE) $)^{12}$ structural and vibrational properties of newly formed $\mathrm{In}_{2} \mathrm{Bi}, \mathrm{In}_{5} \mathrm{Bi}_{3}$ and $\mathrm{InBi}$ compounds on the superconducting properties are unavoidable. To study the superconducting properties of these bimetallic alloys we carried out detailed field and temperature dependent magnetization measurements. Magnetic properties were measured using a Quantum Design MPMS VSM SQUID magnetometer. The transition temperature below which the diamagnetic Meissner effect becomes dominant was confirmed by measuring the zero-field cooled (ZFC) and field-cooled (FC) magnetization. The ZFC is a screening measurement, performed by first cooling the sample in a zero magnetic field to $2 \mathrm{~K}$, subsequently applying a small magnetic field, $\mathrm{H}_{a}=10 \mathrm{Oe}$, and then measuring the resultant magnetization 

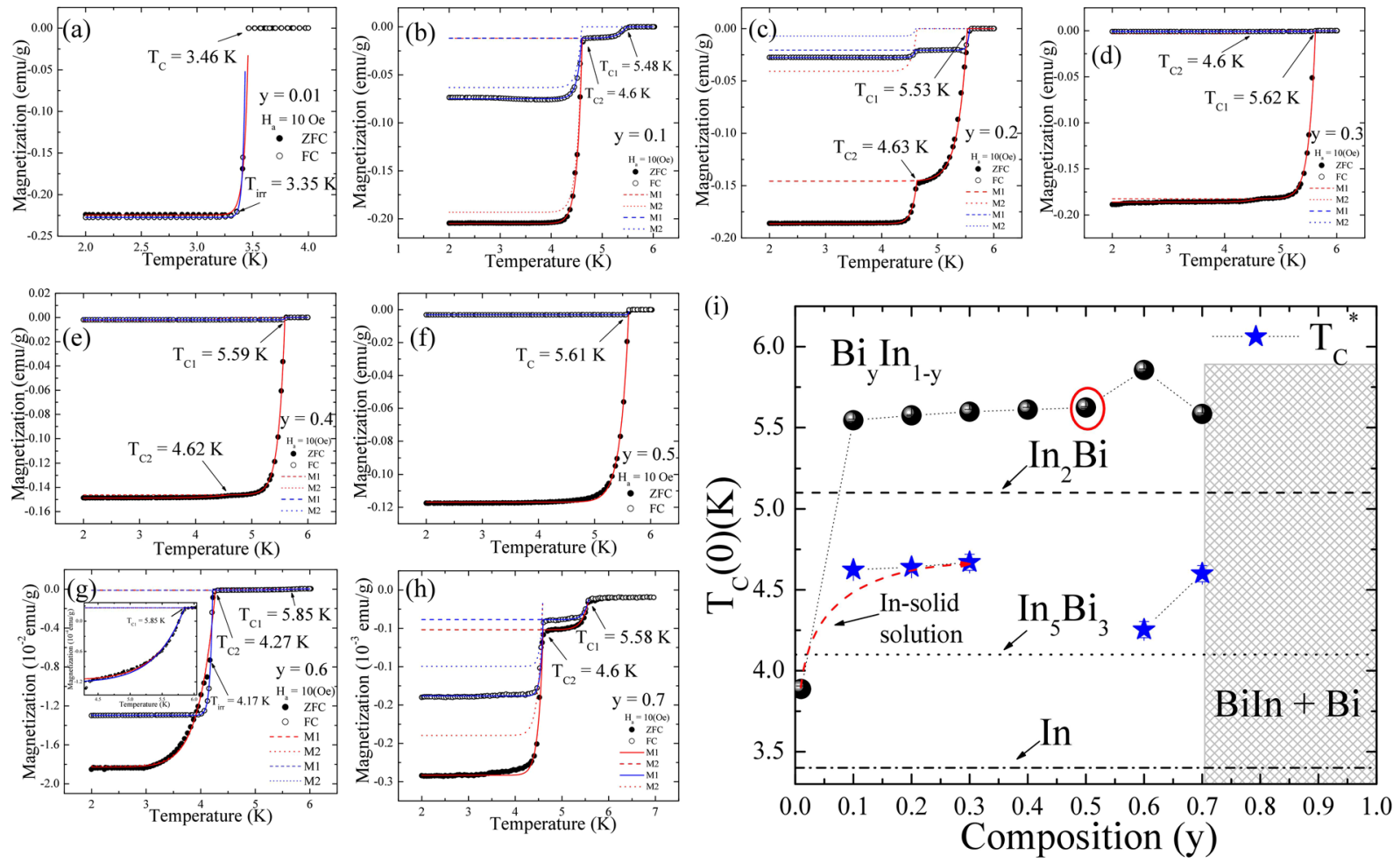

Figure 3. The temperature dependence of $\mathrm{M}(\mathrm{T})$. (a-h) Temperature dependency of magnetization measured between 2 to $6 \mathrm{~K}$ in an external magnetic field of $\mathrm{H}_{a}=10 \mathrm{Oe}$ in the ZFC (shielding) and FC (Meissner) modes. The red and blue solid curve presents the fitted curve of the modified London expression to the data. (i) Fitted value of transition temperature $\mathrm{T}_{\mathrm{C} 1}$ (black sphere) and $\mathrm{T}_{\mathrm{C} 2}$ (blue star) in $\mathrm{H}_{a}=10$ Oe as a function of initial composition.

while warming the sample. The FC is the Meissner flux expulsion measurement, performed by first cooling the sample to $2 \mathrm{~K}$ in a small magnetic field, $\mathrm{H}_{a}=10 \mathrm{Oe}$, and then measuring the resultant magnetization while warming the sample. Four selected samples of field cooled and zero-field cooled $\mathrm{M}(\mathrm{T})$ measurements recorded in the 1.8-6 K range with $\mathrm{H}_{a}=10 \mathrm{Oe}$, as represented in Fig. 3(a-d). As expected, two step-like transitions were observed in Fig. 3(b) due to two immiscible phases that can be described using a superposition of the London equation ${ }^{13}$ for $\operatorname{In}_{2} \mathrm{Bi}(\mathrm{M} 1)$ and $\alpha-\operatorname{In}(\mathrm{M} 2)$, showing two step-like transitions, $\mathrm{T}_{\mathrm{C} 1}$ and $\mathrm{T}_{\mathrm{C} 2}$, which are consistent with the multiphase bismuth-indium system obtained by Hutcherson et al. ${ }^{6}$ and Currie et al. ${ }^{14}$. The step-like magnetization can be well fitted using a modified London equation:

$$
\mathrm{M}(\mathrm{T})=a+H_{a} \times \frac{-1}{4 \pi}\left\{\frac{-3}{2 \rho}\left[1-6\left(\frac{\lambda}{<d>}\right) \operatorname{coth}\left(\frac{<d>}{2 \lambda}\right)+12\left(\frac{\lambda}{<d>}\right)^{2}\right]\right\},
$$

Where $<\mathrm{d}>$ is the mean size, $\rho$ is mass density, and $\lambda_{L}$ is the London penetration depth which varies according to $\lambda_{L}(T)=\lambda_{L}(0)\left[1-\left(T / T_{C}\right)^{P}\right]^{-1 / 2}$. Conventionally, $P=4$ has been utilized to define bulk-like superconductivity for spherical particles. Li et al. ${ }^{15}$ used the above London equation to define the susceptibility in well-separated spherical particles of lead with fix $P=4$. However, the value of $P$ defines only the distribution of the transition temperature, i.e., the higher the value of $P(\geq 1)$ the steeper the distribution of transition temperature will be. In such a scenario, the bulk-like magnetization $\mathrm{M}(\mathrm{T})$, can be well fitted using $P>4$. The solid red and blue lines shown in Fig. 3(a-h) represent the fitted curves for ZFC and FC, respectively, with $M(T)=M_{1}+M_{2}$, and the steepness of $\mathrm{M}(\mathrm{T})$ represented by the value of $P$ and the penetration depth at $\mathrm{H}_{a}=10$ Oe. The fitted values of $\mathrm{T}_{\mathrm{C} 1}(10 \mathrm{Oe})$ and $\mathrm{T}_{\mathrm{C} 2}(10 \mathrm{Oe})$ with respect to the initial composition $\mathrm{y}$ are as shown in Fig. 3(i). The highest value of $\mathrm{T}_{\mathrm{C} 1}=5.85 \mathrm{~K}$ was observed for the $\mathrm{y}=0.6$ sample (and the $\mathrm{T}_{\mathrm{C} 1}$ of $\mathrm{y}=0.7$ is close to that of the $\mathrm{In}_{2} \mathrm{Bi}$ phase), indicating the presence of an $\operatorname{In}_{2} B i$ phase. However, from the XRD data (see Fig. 2(g and h)), we did not observe any sign for $\operatorname{In}_{2} \mathrm{Bi}$ phase, which could be due to the relatively low superconducting volume fraction (SVF) of the $\operatorname{In}_{2} \mathrm{Bi}$ phase, as can be seen from the $\mathrm{M}(\mathrm{T})$ data. Therefore, the above finding shows the potentiality of superconducting measurement even over synchrotron radiation XRD, particularly for superconducting materials. Apparently, enhanced $T_{C}$ was observed for all superconducting phases. The observed enhanced $T_{C}$ for $\operatorname{In}_{2} B i$ and $\operatorname{In}_{5} B i_{3}$, as compared to that of the reported value of the respective single crystal, is because of the stress induced strain effect, as can be seen from the refined SR-XRD data. Hutcherson et al. ${ }^{6}$ carried out a microscopic examination of BiIn eutectic polished samples and revealed a heterogeneous mixture of crystalline grains of $\operatorname{In}_{2} \mathrm{Bi}$ and $\operatorname{In}_{5} \mathrm{Bi}_{3}$ phases. The reported transitions from a eutectic sample obtained at 35 atomic \% of Bi concentration are $5.6 \mathrm{~K}$ and $4.1 \mathrm{~K}$ 


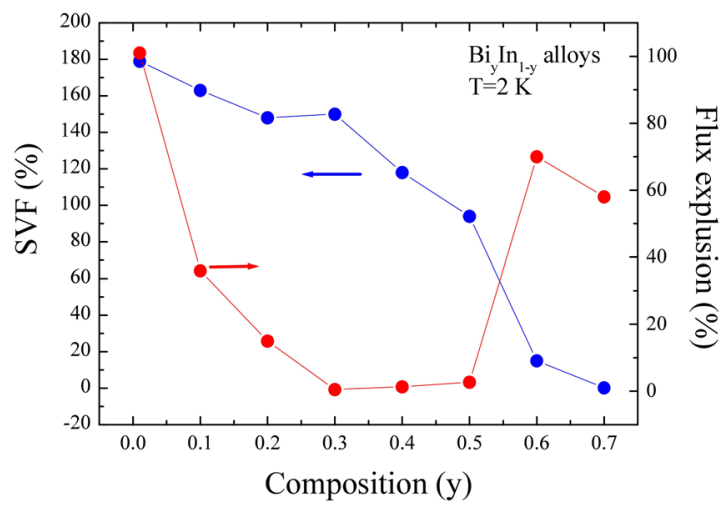

Figure 4. The SVF and flux expulsion analysis. Plot of the calculated values of SVF and flux expulsion with respect to initial composition.

for $\mathrm{In}_{2} \mathrm{Bi}$ and $\mathrm{In}_{5} \mathrm{Bi}_{3}$ phases, respectively. These reported values are somewhat lower, but still in good agreement with our finding. However, for higher concentrations, i.e. 40 to 50 atomic $\%$ of $\mathrm{Bi}$, only transition temperature, $4.1 \mathrm{~K}$, corresponding to $\mathrm{In}_{5} \mathrm{Bi}_{3}$ has been reported which contradicts our observations. Furthermore, the observed enhanced $\mathrm{T}_{\mathrm{C}}$ for an In-Bi solid solution, $\alpha$-In could be the combined effect of enhanced density of state on the Fermi surface and the stress induced strain effect. Since, $\mathrm{T}_{\mathrm{C}}$ is proportional to density of state and Bi has two extra valence electrons compared to In, this results in an increase of density of state $N\left(E_{F}\right)$ on the Fermi surface with an increase of $\mathrm{Bi}$ concentration. The effect of electron and hole doping has been studied in a Tl-Pb-Bi system (which retains the same $f c c-\mathrm{Pb}$ structure for $0.8 \mathrm{Tl}$ to $0.2 \mathrm{Bi}$ concentrations) in which $\mathrm{Tl}$ has one fewer, and $\mathrm{Bi}$ has one more, valence electron than $\mathrm{Pb}$. It has been reported that an increase of $\mathrm{N}\left(\mathrm{E}_{\mathrm{F}}\right)$ from $\mathrm{Tl}$ to $\mathrm{Bi}$ resulted in strong electron-phonon coupling mediated by low lying phonon and showed an enhancement of $\mathrm{T}_{\mathrm{C}}$ from 2.4 to $8 \mathrm{~K}^{16}$. However, the effect of stress induced strain on $\mathrm{T}_{\mathrm{C}}$ cannot be neglected, as samples from $\mathrm{y}=0.1$ to 0.4 are composite in nature. A normal to superconducting transition, namely $\mathrm{T}_{\mathrm{C} 1}$, was observed for the $\mathrm{y}=0.6$ sample due to Josephson coupled with an anisotropic superconducting granular system via inter-granular weak links ${ }^{7}$, as shown in Fig. 3(g). The weak link could be achieved through a normal phase (e.g. InBi and $\mathrm{Bi}$ ) or a superconductor with low $\mathrm{T}_{\mathrm{C}}$ or the grain boundaries. The calculated superconducting volume fraction (SVF) from the ZFC of the pure $\mathrm{In}_{2} \mathrm{Bi}$ phase (Fig. $\left.3(\mathrm{f})\right)$ is $94 \%$, close to the perfect diamagnetism $(-1 / 4 \pi$, according to the CGS system), indicating bulk like superconductivity without considering the demagnetization factor. The calculated low value of flux expulsion (from FC) of $2.7 \%$, indicated strong flux pinning, possibly on the grain boundaries of $\mathrm{In}_{2} \mathrm{Bi}$. The values of SVF and flux expulsion at $2 \mathrm{~K}$ with respect to initial composition are as shown in the inset of Fig. 4 . The high value of flux expulsion and relatively low value of SVF in superconducting-normal phase composites, $y=0.6$ and 0.7 , suggest relatively strong-linked grains in these samples. The SVF exceeding $100 \%$ in composite $y=0.4$ to 0.1 samples is attributed to the magnetization contribution from the two superconducting phases and the demagnetization factor of the sample. Due to the high density of defects and strong flux pinning, a very low value of flux expulsion was observed for these composites. As can be seen from Fig. 3(a), the FC and ZFC curve of $y=0.01$, $\alpha$-In, overlaps below $\mathrm{T}_{\mathrm{C}}(10 \mathrm{Oe})=3.46 \mathrm{~K}$ down to lowest available temperature, which reflects reversible behavior due to weaker pinning and/or a lower density of defects. The calculated high value of SVF, $179 \%$ is attributed to the demagnetization effect of the sample. As expected, $101 \%$ of flux expulsion indicated an ideal, pinning-free bulk sample, with the sample always remaining in thermodynamic equilibrium. The similar reversible region was also observed for the $\operatorname{In}_{2} \mathrm{Bi}$ phase of $\mathrm{y}=0.1$ and $\mathrm{y}=0.6$ samples, suggesting that both grain coupling and intra-grain pinning are relatively poor. As we can see, the magnetization SVF for the $\mathrm{In}_{2} \mathrm{Bi}$ phase in the former and later samples is only $\sim 9 \%$ and $\sim 0.09 \%$, respectively. Furthermore, the reversibility in the $y=0.6$ composite could be due to strain caused by normal vortex cores embedded in the superconducting phase. According to Kogan et al. ${ }^{17}$ the strains in this type of composite system arise due to the difference in density of the normal (i.e. $\mathrm{InBi}$ ) and superconducting (i.e. $\operatorname{In}_{2} \mathrm{Bi}$ and $\operatorname{In}_{5} \mathrm{Bi}_{3}$ ) phases, which are related to the stress dependency of the critical temperature.

Isothermal Magnetization and theoretical analysis. The observed overlapping and strong flux pinning from $\mathrm{M}(\mathrm{T})$ was further confirmed from the isothermal magnetization $\mathrm{M}\left(\mathrm{H}_{a}\right)$ measured at $2 \mathrm{~K}$, as shown in Fig. 5(a-h). The observed pronounced reversibility for $\mathrm{y}=0.01, \alpha$-In is in excellent agreement with the overlapping of FC and ZFC. The rounded shape of the $\mathrm{M}\left(\mathrm{H}_{a}\right)$ near penetration field $\mathrm{Hp}$ is plainly due to large local demagnetization at the corners ${ }^{18}$. Similar reversible behavior was also spotted for $\mathrm{y}=0.1-0.2$ and 0.6 composite samples above irreversible field $\mathrm{H}_{\text {irr }}$ as shown in Fig. 5(b, c) and (g). The field $\mathrm{H}_{\text {irr }}$ defines the transition line in the $\mathrm{M}\left(\mathrm{H}_{a}\right)$ that divides the intermediated regions into reversible and irreversible regions of flux configuration. The observed irreversibility in the intermediate state for all the samples could be because of differences in topologies upon flux entry and exit, as reported for type-I superconductors ${ }^{19}$. The difference in the shape of the $\mathrm{M}\left(\mathrm{H}_{a}\right)$ hysteresis of all the samples reflects how strongly the intermediate state and reversible-irreversible transition depends on grain coupling, intra-grain pinning and density of defects. For type-II superconductors, the magnetization can be defined as $m=-h /(1-N)$, where $N$ is the demagnetization factor, which can vary with the direction of an external field relative to the principle axis of the superconductor, $\mathrm{m}\left(\mathrm{H}_{a}\right)=\mathrm{M}\left(\mathrm{H}_{a}\right) / \mathrm{M}\left(\mathrm{H}_{\mathrm{C} 1}\right)$ and $\mathrm{h}=\left(\mathrm{H}_{a} / \mathrm{H}_{\mathrm{C} 2}\right)$. 

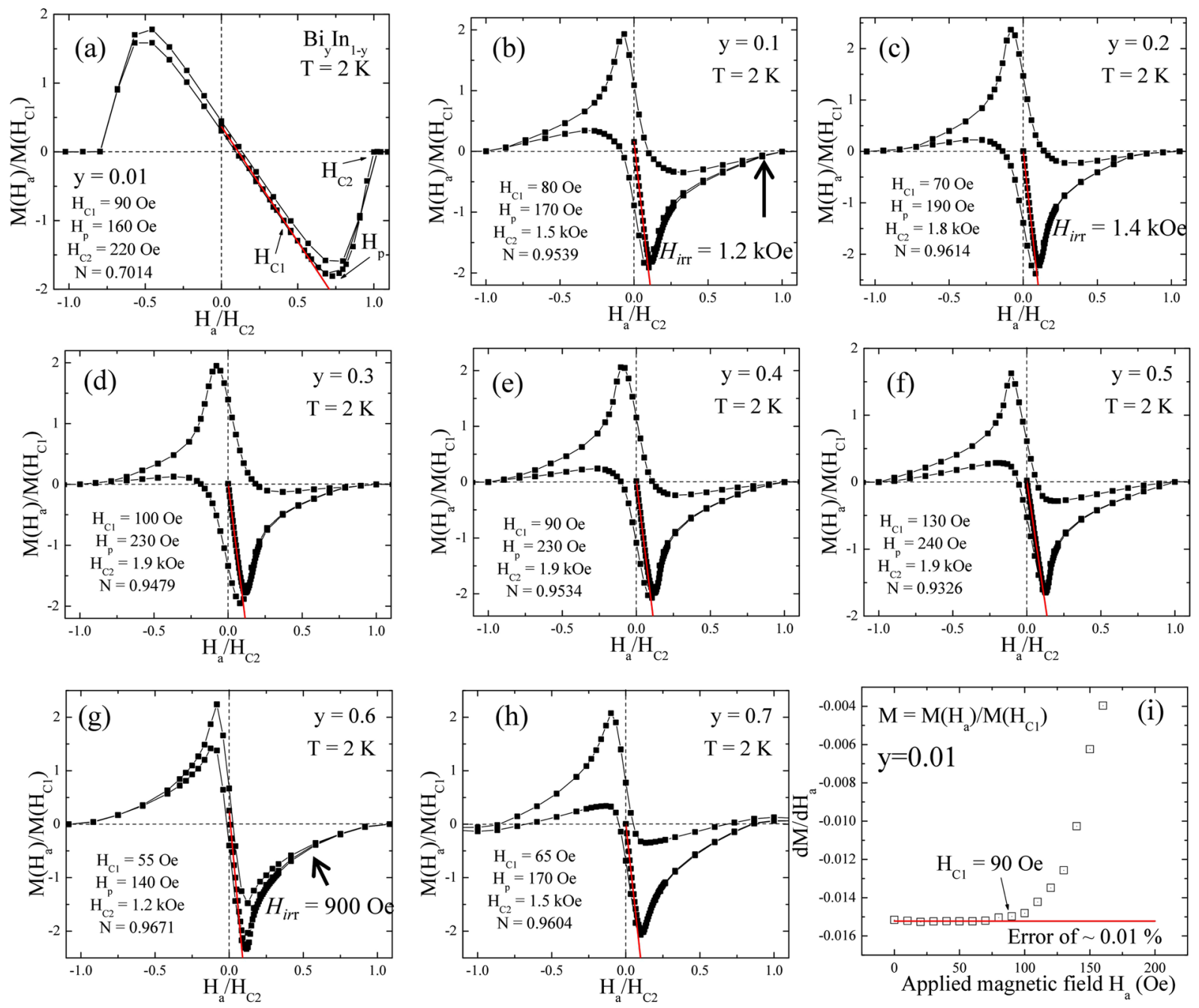

Figure 5. The applied magnetic field dependence of $\mathrm{M}\left(\mathrm{H}_{a}\right)$. (a-h) Isothermal magnetization measured at $2 \mathrm{~K}$ where the reduced magnetization is defined as the ratio of magnetization $\mathrm{M}\left(\mathrm{H}_{a}\right)$ to the absolute value at the first critical field $\mathrm{M}\left(\mathrm{H}_{\mathrm{C} 1}\right)$. The red solid line is the linear fit to the first magnetization curve. The observed irreversible field is marked by arrows. (i) represents the differential of the first magnetization curve and the linear fit to it.

The $\mathrm{M}\left(\mathrm{H}_{\mathrm{C} 1}\right)$ is the magnetization at lower critical field $\mathrm{H}_{\mathrm{Cl}}$, below which the superconductor retains the perfect Meissner effect, and $\mathrm{H}_{\mathrm{C} 2}$ is the upper critical field above which the superconductor behaves as normal conductor. The deviation of magnetization in the low field region of linearity gives the value of $\mathrm{H}_{\mathrm{Cl}}{ }^{20}$. The solid red lines in Fig. 5(a-h) represent the linear fit to $\mathrm{M}\left(\mathrm{H}_{a}\right)$. The goodness of linearity fit in the low field region can be represented by differential ${ }^{21}$, as shown in Fig. 5(i). In general, the obtained $\mathrm{H}_{\mathrm{Cl}}$ in $\mathrm{M}\left(\mathrm{H}_{a}\right)$ measurement suffers not only from demagnetization factor but also surface barrier effect. The type-II like hysteresis loop $\mathrm{M}\left(\mathrm{H}_{a}\right)$ then reflects the characteristic of the penetration of magnetic flux into the alloy. As can be seen in Fig. 5(e), the $\mathrm{M}\left(\mathrm{H}_{a}\right)$ loop displays an asymmetric profile in the field-increasing and -decreasing branches and a large penetration field $\mathrm{Hp} \sim$ 230 Oe was obtained at $\mathrm{T}=2 \mathrm{~K}$ for $\mathrm{y}=0.4$ sample. The irreversibility of the entry and exit of magnetic flux lines through the alloys surfaces shows that it is mainly the geometric edge barriers that control the movements of flux lines at the surface; a Bean-Livingston ${ }^{22}$ surface barrier effect has been reported in high $\mathrm{T}_{\mathrm{C}}$ superconductors ${ }^{23}$. It should be noted that the determination of $\mathrm{H}_{\mathrm{C} 1}$ is of primary importance since it allows one to extract the magnetic penetration depth fundamental parameter characterizing the superconducting condensate and carrying information about the underlying pairing mechanism. A popular approach to measure $\mathrm{H}_{\mathrm{C} 1}$ consists of measuring the magnetization $\mathrm{M}\left(\mathrm{H}_{a}\right)$ as a function of $\mathrm{H}_{a}$ and then identify the deviation of the linear Meissner response which would correspond to the vortex penetration. One may argue that the $\mathrm{H}_{\mathrm{C} 1}$ values obtained from the deviation of magnetization in the low field region may not reflect the true $\mathrm{H}_{\mathrm{C} 1}$ but the flux entry field because of the BeanLivingston surface barrier ${ }^{22}$. However, it is clear that the influence of surface barrier is important in our investigated alloys system since: (i) the magnetic hysteresis loops are asymmetric close to $\mathrm{T}_{\mathrm{C}}$. (ii) Therefore, if the surface barrier should be taken into account, the true $\mathrm{H}_{\mathrm{C} 1}$ would be much smaller than the obtained value from the deviation of magnetization in the low field region. The upper critical field $\mathrm{H}_{\mathrm{C} 2}$ is defined as the field $\mathrm{H}_{a}$ at which the magnetization $\mathrm{M}\left(\mathrm{H}_{a}\right)$ shows zero value. Except for $\mathrm{y}=0.01, \alpha$-In, the value of $N$ is close to $\sim 0.95(1)$, indicating 


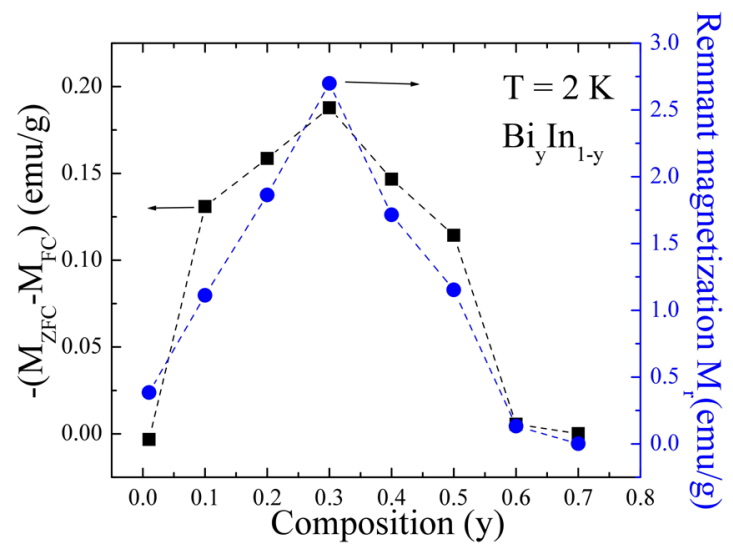

Figure 6. The initial composition dependence of $\left(\mathrm{M}_{\mathrm{ZFC}}-\mathrm{M}_{\mathrm{FC}}\right)$ and $\mathrm{M}_{\mathrm{r}}$. Plot of the absolute value of $\left(\mathrm{M}_{\mathrm{ZFC}}-\mathrm{M}_{\mathrm{FC}}\right)$ (black square) and remnant magnetization $\mathrm{M}_{\mathrm{r}}$ (blue circles) as a function of initial composition.

superconducting lasting over an infinite range with $\mathrm{H}_{a}$ applied perpendicular to the surface. This accordance reveals that the coupling between the observed spherical grains from the SEM images extends over infinity. Thus, the demagnetization effect is governed by the outward appearance of the sample, not by the size and shape of each grain. In other words, as discussed before, the sample shows bulk-like superconductivity due to weakly-connected grains, even though the grain boundaries exist. Furthermore, Fig. 6 represents the calculated remnant value at zero field $\mathrm{M}_{\mathrm{r}}(0)$ and gives an idea about the amount of trapped flux which can be correlated with $-\left(\mathrm{M}_{\mathrm{ZFC}}-\mathrm{M}_{\mathrm{FC}}\right)$ referring to the corresponding maximum value of flux excluded in the Meissner state. The highest value of $M_{r}(0)$ is for $\mathrm{y}=0.3$ composites, suggesting a larger capability to trap flux. The calculated values of $\mathrm{H}_{\mathrm{C} 1}, \mathrm{H}_{\mathrm{C} 2}$, penetration field $\mathrm{Hp}$ and demagnetization factor $\mathrm{N}$, remnant magnetization $\mathrm{M}_{\mathrm{r}},-\left(\mathrm{M}_{\mathrm{ZFC}}-\mathrm{M}_{\mathrm{FC}}\right)$, SVF and flux expulsion $f_{e x}(\%)$ at $2 \mathrm{~K}$ for all the samples are as tabulated in the supplementary information of Table S2.

To estimate the thermal effect of the superconducting properties of a $\mathrm{Bi}_{\mathrm{y}} \mathrm{In}_{1-\mathrm{y}}$ system, $\mathrm{M}\left(\mathrm{H}_{a}\right)$ loop measurement of all the samples $(y=0.01$ to 0.7$)$ was carried out at various temperatures below the transition temperature, as can be seen in Figure S2 (see the supporting information: Figure S2). Following a similar procedure as mentioned before, the values of $\mathrm{H}_{\mathrm{C} 1}$ and $\mathrm{H}_{\mathrm{C} 2}$ were estimated at various temperatures and the observed values plotted as shown in Fig. $7(\mathrm{a}$ and $\mathrm{b})$. The $\mathrm{H}_{\mathrm{C} 1}$ and $\mathrm{H}_{\mathrm{C} 2}$ can be fitted with a power law, in accordance with $H_{C 1}(T)=H_{C 1}(0)\left(1-\beta\left(T / T_{C 1}(0)\right)^{\alpha}\right)$ and $H_{C 2}(T)=H_{C 2}(0)\left(1-\delta\left(T / T_{C 1}(0)\right)^{\alpha}\right)$, where $\beta$ and $\delta$ are the fitting parameters with a value close to $\sim 1(0.1)$, and $\mathrm{H}_{\mathrm{C} 1}(0)$ and $\mathrm{H}_{\mathrm{C} 2}(0)$ are the lower and upper critical field at zero temperature, respectively (see in the supplementary information of Table S2). The value of $\alpha$ represents the coupling strength obtained from the $\alpha$-model (which will be discussed later in the text) and it was kept fixed for respective samples. The resulting fitted values of $\mathrm{H}_{\mathrm{C} 1}(0)$ and $\mathrm{H}_{\mathrm{C} 2}(0)$ allow us to calculate the coherence length $\xi_{G L}(0)$ and the penetration depth $\lambda(0)$ using a Ginzburg-Landau (GL) formulation ${ }^{24}, \xi_{G L}(0)=\sqrt{\Phi_{0} / 2 \pi H_{C 2}(0)}$ and $\lambda(0)=\sqrt{\Phi_{0} / 2 \pi H_{C 1}(0)}$, where $\Phi_{o}=\frac{\mathrm{h}}{2 \mathrm{e}}=2.0678 \times 10^{9}$ Oe $A^{2}$ is the quantum flux ${ }^{25}$, which yields minimum $\xi_{G L}(0) \sim 40.1 \mathrm{~nm}(\mathrm{y}=0.4)$ and maximum $\lambda(0) \sim 220 \mathrm{~nm}(\mathrm{y}=0.7)$ at $0 \mathrm{~K}$. Using the relation $\kappa(0)=\lambda(0) / \xi_{G L}(0)$ we calculated the value $\kappa(0)$, which varied from $1.6(\mathrm{y}=0.01)$ to $5.0(\mathrm{y}=0.7)$, indicating type II superconducting behavior. In addition, the thermodynamic critical field, $H_{T C}(0) \approx \sqrt{H_{C 1}(0) H_{C 2}(0)}$ was calculated, showing a maximum value of $\sim 534 \mathrm{Oe}$ for the $\operatorname{In}_{2} \mathrm{Bi}$ superconductor $(\mathrm{y}=0.5)$. The estimated superconducting parameters for all the BiIn samples are as tabulated in the supplementary information of Table S3.

Applied magnetic field dependency of ZFC and FC Magnetization. The cause behind an enhanced transition temperature of $\operatorname{In}_{2} \mathrm{Bi}$ and $\operatorname{In}_{5} \mathrm{Bi}_{3}$ has not been clearly identified yet. There are a few possibilities, such as internal strain or short range disorder, as reported for an InSn system, or the enhanced electron-phonon coupling mediated by low lying phonons, as observed for a $\mathrm{BiPb}$ system. The effect due to a short range disorder can be avoided, as the calculated mean free path at $2 \mathrm{~K}$ is a multiple of lattice constants. From GL theory, the carrier mean free path, $\ell$, at low temperature $\mathrm{T}=2 \mathrm{~K}$ can be estimated using

$$
\frac{1}{\ell}=\frac{\xi_{o}}{0.882}\left[\frac{0.546}{\xi_{G L}^{2}(0)\left(1-T / T_{C}\right)}-\frac{1}{\xi^{2}(0)}\right],
$$

where $\xi(0)=3640 \AA$ is the BCS coherence length of bulk $\operatorname{In}^{26}$. To estimate the superconducting coupling strength, we carried out ZFC measurement at various external applied field $\mathrm{H}_{a}$, as shown in Figure S3 (see the supporting information: Figure S3). The Meissner diamagnetic screening signal appears to shift consistently to a lower temperature as $\mathrm{H}_{a}$ is increased. The relatively sharp distribution of the transition temperature from the $\mathrm{y}=0.01$ sample, as compared to other superconductors, signals that $\alpha$-In remains in the Meissner state below 150 Oe. The results are in good agreement with the estimated lower critical field $\mathrm{H}_{\mathrm{Cl}}(0)=134$ (3) Oe for $\alpha$-In and the maximum $\mathrm{H}_{\mathrm{C} 1}(0)=106(5)$ Oe for the remaining samples. A broad distribution of transition temperatures, extending to the lowest available temperature at field $\mathrm{H}_{a}>100 \mathrm{Oe}$, was observed for sample $\mathrm{y}=0.1$ to 0.7 . Schuck et al. from 

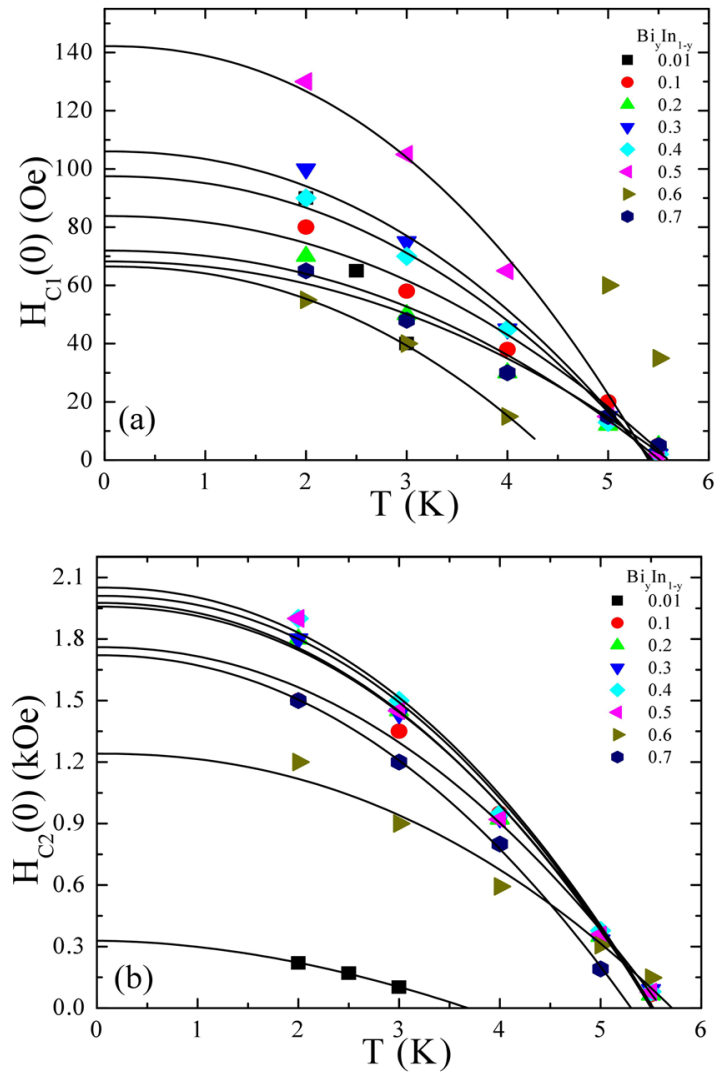

Figure 7. The temperature dependence of $\mathrm{H}_{\mathrm{C} 1}(0)$ and $\mathrm{H}_{\mathrm{C} 2}(0)$. (a,b) Lower and upper critical field as a function of temperature. Solid lines are fit to the power law mentioned in the text.

single crystal $\mathrm{KOs}_{2} \mathrm{O}_{6}$ and Rogacki et al. ${ }^{27,28}$ from single crystal $\mathrm{RbOs}_{2} \mathrm{O}_{6}$ reported the observation of similar broad distributions of transition temperatures to what was observed in the InBi system. However, they correlated the broad distribution of transition temperatures with weak-pinning behavior, which is contradictory to a BiIn system. The observed broad distribution could be the sign of a weakly-linked granular superconductor, as these BiIn superconductors include both weak and strong-pinned superconductors. The fitted value of $P$ to the ZFC of all the superconducting samples within the Meissner state is higher than 4, whereas in the flux pinning state it lies between 1 to 2 . Therefore, values of $P$ can help in defining the state of the linked intergranular superconducting system. The fitted values of $P\left(H_{a}\right)$ and the London penetration depth $\lambda\left(\mathrm{H}_{a}\right)$ are as plotted in Fig. $8(\mathrm{a}$ and b), respectively. Within a Meissner state $\lambda\left(\mathrm{H}_{a}\right)$ remains constant or shows slightly decreasing behavior with an increase of $\mathrm{H}_{a}\left(\leq \mathrm{H}_{\mathrm{Cl}}(0)\right)$. The reduction of the diamagnetic screening in the flux pinning state is known to be caused by the increase of $\lambda$ with $\mathrm{H}_{a}$, which in turn reduces the SVF, hence the shielding capability of a superconductor ${ }^{29}$. Furthermore, the suppression of critical temperature with an increase of field $\mathrm{H}_{a}$ can be well described using $T_{C}\left(H_{a}\right)=T_{C}(0)\left[1-H_{a} / H_{c}(0)\right]^{\gamma}$, where $T_{C}(0)$ is the zero field critical temperature, $\mathrm{H}_{\mathrm{C}}(0)$ is the zero temperature critical field, and $\gamma$ is a fitting parameter, as shown in Fig. 9(a). All the fitting parameters are as tabulated in the supplementary information of Table S4. The fitted value of $\mathrm{H}_{\mathrm{C}}(0)$ for each sample is close to the $\mathrm{H}_{\mathrm{C} 2}(0)$ and the maximum value is $\sim 6.5$ times that of the pure Indium phase. Padamsee et al. ${ }^{30}$ introduced the so-called $\alpha$-model, by which the relative coupling strength, $\alpha=\Delta(0) / k_{B} T_{C}$ (where, $\Delta(0)$ is the superconducting energy gap at $0 \mathrm{~K}$ and $\mathrm{k}_{\mathrm{B}}$ is the Boltzmann constant) of a superconducting system can be revealed in the deviation of $\mathrm{H}_{\mathrm{C}}(\mathrm{T}) / \mathrm{H}_{\mathrm{C}}(0)$ from the parabolic dependency of $1-\left(T / T_{C}\right)^{2}$. It is known that weak-coupled systems yield negative deviations, while strong-coupled systems yield positive deviations ${ }^{31}$. The observed negative deviation for $\alpha$-In $(\mathrm{y}=0.01)$ and the positive deviation for the remaining BiIn superconductor $(\mathrm{y}=0.1$ to 0.7$)$ can be fitted by using $\frac{H_{C}(T)}{H_{C}(0)}=\left[1-\left(\frac{T}{T_{C}}\right)^{\alpha}\right]$, as shown in Fig. 9(b). The solid line represents the fitted curve, and the fitted values of $\alpha$ are as shown in the supplementary information of Table S4. A minimum of $\alpha=1.835$ (2) (close to weak-coupled bulk In, 1.9) from $y=0.01$ and a maximum of 2.231(9) (larger than strong-coupled bulk Pb) from $\mathrm{y}=0.5$, indicate the pure $\mathrm{In}_{2} \mathrm{Bi}$ phase is larger than BCS prediction of 1.764 . The fitted values of the coupling strength for $\mathrm{In}_{2} \mathrm{Bi}$ superconductor could be mediated by low lying phonons resulting in an enhanced $\mathrm{T}_{\mathrm{C}}$. The average value of logarithmic phonon energy $\omega_{\ln }$ and the dimensionless electron-phonon coupling constant $\lambda_{\text {ep }}$ for a sample of BiIn can be calculated from the Eliashberg theory-based Allen-Dynes formulation ${ }^{2}$. The corrections of the BCS values by strong electron-phonon interactions have been deduced in the following approximate analytic formulas that link $\omega_{\mathrm{ln}} / \mathrm{T}_{\mathrm{C}}$ to experimental thermodynamic quantities: 

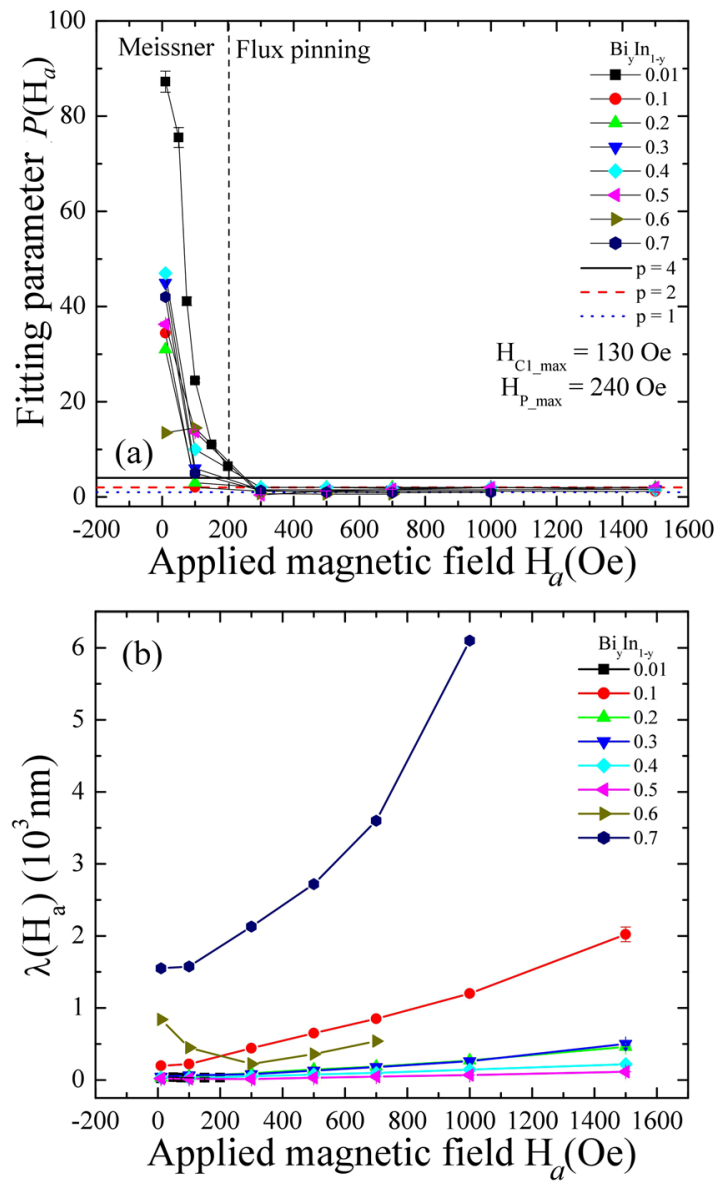

Figure 8. The applied magnetic field dependence of $P\left(\mathrm{H}_{a}\right)$ and $\lambda\left(\mathrm{H}_{a}\right)$. Applied magnetic field dependence of the fitted values of $(\mathbf{a}) P\left(\mathrm{H}_{a}\right)$ and $(\mathbf{b})$ London penetration depth $\lambda\left(\mathrm{H}_{a}\right)$, respectively.

$$
\alpha=\frac{\Delta(0)}{k_{B} T_{C}(0)}=1.764\left[1+12.5\left(T_{C}(0) / \omega_{l n}\right)^{2} \times \ln \left(\omega_{l n} / 2 T_{C}(0)\right)\right]
$$

The calculated values of $\omega_{\ln }$ are as shown in Table $S 4$, and we see that a minimum of $45.92 \mathrm{~K}$ was calculated for the pure $\operatorname{In}_{2} \mathrm{Bi}$ sample $(\mathrm{y}=0.5)$. Next, the dimensionless $\lambda_{\mathrm{ep}}$ can be estimated from McMillan's formulation ${ }^{32}$. However, as can be seen from Fig. 10, the McMillan formulation is useful only for intermediate coupled superconductors, $\lambda_{\text {ep }}<1$. As shown in Fig. 10, most of the phonon mediated superconductors can be well described using Allen and Dynes' modified McMillan formula ${ }^{2}$,

$$
\frac{T_{C}}{\omega_{l n}}=\frac{f_{1} f_{2}}{1.2} \exp \left[\frac{-1.04\left(1+\lambda_{e p}\right)}{\lambda_{e p}-\mu^{*}\left(1+0.62 \lambda_{e p}\right)}\right]
$$

where $f_{1}=\left[1+\left(\frac{\lambda_{e p}}{\Lambda_{1}}\right)^{3 / 2}\right]^{1 / 3}$ is a strong coupling correction function and $f_{2}=1+\left(\frac{\varpi_{2}}{\omega_{l n}}-1\right) \lambda_{e p}^{2} /\left(\lambda_{e p}^{2}+\Lambda_{2}^{2}\right)$ is a shape correction function. Where $\Lambda_{1}=2.46\left(1+3.8 \mu^{*}\right), \Lambda_{2}=1.82\left(1+6.3 \mu^{*}\right)\left(\frac{\varpi_{2}}{\omega_{l n}}\right)$ and $\varpi_{2}=\sqrt{<\omega_{l n}^{2}>}$ is the square root average logarithm of the phonon frequency introduced by Allen and Dynes, and $\mu^{*}$ is the Coulomb pseudo potential. The $\mu^{*}$ describes the Coulomb pseudopotential to represent the repulsive part of the pairing interaction, and to calculate $\lambda_{\text {ep }}$ we set $\mu^{*}=0.11$ and $\varpi_{2}=1.3 \omega_{l n}$. The plot of $\mathrm{T}_{\mathrm{C}} / \omega_{\mathrm{ln}}$ vs. $\lambda_{\text {ep }}$ of the BiIn system (red filled circle) along with different phonon mediated superconductors is as shown in Fig. 10. The blue solid line represents the calculated $T_{C} / \omega_{l n}$ using Allen and Dyne's formula. For comparison, we have also shown the results from the BCS and McMillan formulation (black and orange solid lines, respectively). From the above Fig. 10, for $\lambda_{\text {ep }}<1$, for the Allen and Dyne formulation reduced to McMillan formulation ${ }^{32}$ and for $\lambda_{\text {ep }}<0.2$, both the Allen-Dyne and McMillan formulations are reduced to the BCS prediction ${ }^{33}, 34$. The calculated value of $\lambda_{\text {ep }}=1.453, \omega_{\ln }=45.92 \mathrm{~K}$ for BiIn is in good agreement with the earlier reported value of $\lambda_{\mathrm{ep}}=1.40, \omega_{\ln }=46 \mathrm{~K}^{2}$. However, using McMillan's formula with $\mu^{*}=0.11$ and assuming $\omega_{\ln }$ to be same as a Debye frequency, we found that the ratio $\mathrm{T}_{\mathrm{C}} / \omega_{\mathrm{ln}}$ for $\operatorname{In}_{2} \mathrm{Bi}$ is reproduced with relatively high $\lambda_{\mathrm{ep}} \sim 1.68$, which contradicts previous findings. In 

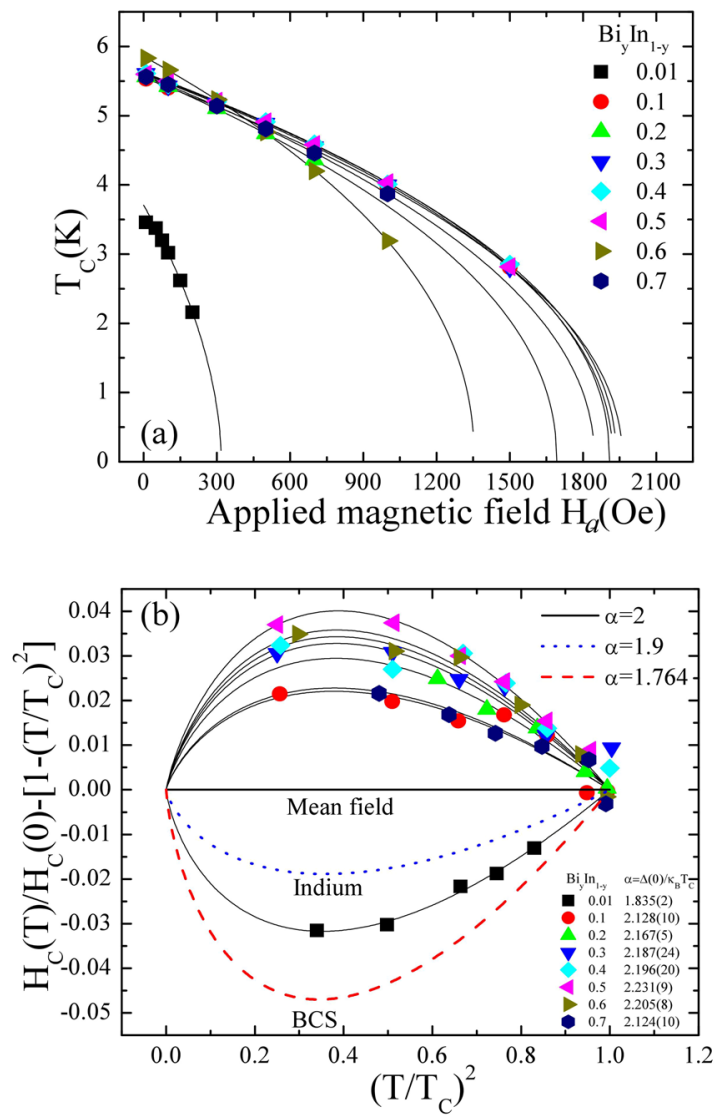

Figure 9. The applied magnetic field dependence of $\mathrm{T}_{\mathrm{C}}\left(\mathrm{H}_{a}\right)$ and coupling strength analysis. (a) The effect of applied field $\mathrm{H}_{a}$ on the transition temperature $\mathrm{T}_{\mathrm{C}}$ of BiIn samples. The solid line is fitted to the expression given in the text. (b) Deviation of $\mathrm{H}_{\mathrm{C}}(\mathrm{T}) / \mathrm{H}_{\mathrm{C}}(0)$ from the parabolic dependency of $1-\left(\mathrm{T} / \mathrm{T}_{\mathrm{C}}\right)^{2}$ fitted to the expression $H_{C}(T) / H_{C}(0)=\left[1-\left(T / T_{C}\right)^{\alpha}\right]$, where $\alpha$ represents the coupling strength of the superconductor.

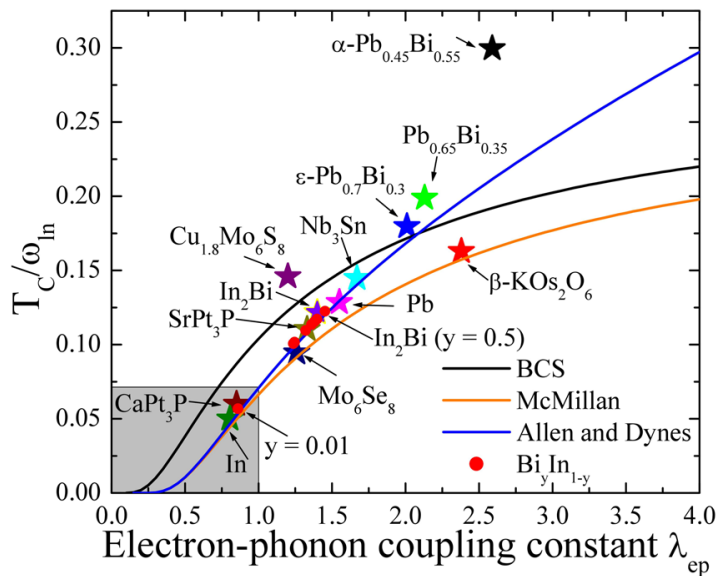

Figure 10. The dependence of $\mathrm{T}_{C} / \omega_{\ln }$ on $\lambda_{\text {ep }}$. The plot of $\mathrm{T}_{\mathrm{C}} / \omega_{\ln }$ as a function of the electron-phonon coupling constant $\lambda_{\text {ep }}$ of BiIn (red circles) calculated from the Allen and Dynes modified McMillan formula (blue solid line). The black and orange colored solid lines represent calculated $\mathrm{T}_{C} / \omega_{\ln }$ using BCS and McMillan's formula. For comparison, the different phonon mediated superconductors (stars) are also shown.

order to match the experimental value of $\mathrm{T}_{\mathrm{C}}$ the required $\lambda$ would be 1.2-1.4 (with reasonable Coloumb pseudo potential $\mu^{*}$ of $0.06-0.09$ ), reflecting the uncertainty in the estimation of Coloumb pseudo potential. It is known that disorder/substitution can affect the Coulomb pseudo potential in superconductor ${ }^{35}$. Hence we could infer that the local inhomogeneity introduced by disorder/substitution in bimetallic BiIn alloys may play nontrivial 
role in the influence of Coulomb pseudo potential. Indeed, the intimate relationship between local inhomogeneity and superconductivity has been extensively studied in the context of high $\mathrm{T}_{\mathrm{C}}$ superconductors, and the possible enhancement of superconductivity by local inhomogeneities has been discussed recently by Martin et al. ${ }^{36}$ in the weak coupling BCS regime.

From Fig. 10, we can conclude that enhanced $\mathrm{T}_{\mathrm{C}}$ is the combined effect of phonon energy and the electron-phonon coupling. For example, $\varepsilon-\mathrm{Pb}_{0.7} \mathrm{Bi}_{0.3}\left(\lambda_{\text {ep }} \sim 2.01, \omega_{\text {ln }}=47 \mathrm{~K}\right)$ and $\mathrm{Pb}_{0.8} \mathrm{Bi}_{0.2}\left(\lambda_{\text {ep }} \sim 1.88, \omega_{\text {ln }}=46 \mathrm{~K}\right)$ have the same phonon energy as $\mathrm{In}_{2} \mathrm{Bi}$, but higher values of $\lambda_{\mathrm{ep}}$, resulting in enhanced Tc of $8.45 \mathrm{~K}$ and $7.95 \mathrm{~K}$, respectively ${ }^{2}$. Similarly, $\mathrm{Mo}_{6} \mathrm{Se}_{8}\left(\lambda_{\mathrm{ep}}=1.27, \omega_{\mathrm{ln}}=70 \mathrm{~K}\right)$ and $\mathrm{SrPt}_{3} \mathrm{P}\left(\lambda_{\mathrm{ep}}=1.33, \omega_{\mathrm{ln}}=77 \mathrm{~K}\right)$ which overlap InBi superconductors, as shown in the figure, also show enhanced $\mathrm{T}_{\mathrm{C}}$ of 8.5 and $6.34 \mathrm{~K}$, respectively ${ }^{3,4}$. Since, $\lambda_{\text {ep }}$ for these two superconductors is lower than $\operatorname{In}_{2} \mathrm{Bi}$, but with high value of phonon energy. Moreover, there are a few superconductors, as shown in the figure, which are beyond Allen and Dynes' prediction, and therefore more theoretical work needs to be carried out. It is known that disorder, substitution in weak coupling superconductivity could enhance the electron-phonon coupling constant. A simplified physical model of electron-phonon coupling has been developed by Gao et al. ${ }^{37}$ to allow heat transfer from phonons to electrons, and applied to study defect or disorder as a function of the strength of electron-phonon coupling. The number of point defects produced in the primary damage state increases with increasing strength of electron-phonon coupling, implying the defect or disorder/substitution plays a role in the increasing strength of electron-phonon coupling constant. As reported in $\mathrm{Cu}_{x} \mathrm{Bi}_{2} \mathrm{Se}_{3} \mathrm{~F}$ superconductor by Zhang et al. ${ }^{35}$, first principle calculation was introduced to investigate the role of electron-phonon coupling for the superconducting pairing in the prime candidate $\mathrm{Cu}_{x} \mathrm{Bi}_{2} \mathrm{Se}_{3}$. In their comprehensive analysis reveal that electron-phonon scattering process in this system is dominated by zone center and boundary optical modes, with coexistence of phonon stiffening and softening, suggesting that superconductivity may not only come from pure electron-phonon coupling but also disorder effect. In the present work, we also cannot ignore the possible enhancement of electron-phonon coupling by local inhomogeneity introduced by doping defect or disorder/substitution in bimetallic BiIn alloys. From the above finding, it's clear that the observed enhanced $\mathrm{T}_{\mathrm{C}}$ for $\mathrm{a} \mathrm{Bi}_{\mathrm{y}} \mathrm{In}_{1-\mathrm{y}}$ system (i.e. $\mathrm{In}_{2} \mathrm{Bi}$ and $\mathrm{In}_{5} \mathrm{Bi}_{3}$ ) is the effect of strong electron-phonon coupling to low-lying phonons. The effect of low lying phonons can be further proved by carrying out electron-tunneling spectroscopy measurements.

\section{Discussion}

The strong-coupling superconducting behavior mediated by low lying phonons has been reported to be the cause for enhanced $\mathrm{T}_{\mathrm{C}}$ in $\mathrm{Bi}_{\mathrm{y}} \mathrm{In}_{1-\mathrm{y}}$ superconductors. The maximum strong-coupling superconducting strength, $\alpha=2.231(9)$, phonon energy, $\omega_{\mathrm{ln}}=45.9 \mathrm{~K}$, electron-phonon coupling constant, $\lambda_{\mathrm{ep}}=1.453$, and critical field, $\mathrm{H}_{\mathrm{C}}(0)=1909$ (3) Oe has been estimated from pure $\operatorname{In}_{2} \mathrm{Bi}(\mathrm{y}=0.5)$ samples using Allen and Dynes' formulation. The different superconducting parameters estimated using GL formulation show $\mathrm{Bi}_{\mathrm{y}} \mathrm{In}_{1-\mathrm{y}}$ is a type-II superconductor with GL constant, $1.6 \leq \mathrm{k}(0) \leq 5$ for $0.01 \leq \mathrm{y} \leq 0.7$. The room temperature SR-XRD refinement carried out by using weighted analysis and superconducting measurement reveals the formation of both pure $\alpha$-In $(y=0.01), \operatorname{In}_{2} \mathrm{Bi}(\mathrm{y}=0.5)$ and composite $\alpha-\operatorname{In}+\operatorname{In}_{2} \mathrm{Bi}(\mathrm{y}=0.1-0.4), \operatorname{In}_{2} \mathrm{Bi}+\operatorname{In}_{5} \mathrm{Bi}_{3}+\operatorname{InBi}(\mathrm{y}=0.6-0.7)$ samples, respectively. The ZFC-FC temperature dependent measurement revealed two-gap superconductivity in $\mathrm{y}=0.1-$ $0.4\left(\alpha-\mathrm{In}+\mathrm{In}_{2} \mathrm{Bi}\right)$ and $0.6-0.7\left(\mathrm{In}_{2} \mathrm{Bi}+\operatorname{In}_{5} \mathrm{Bi}_{3}\right)$ and a single gap in $\mathrm{y}=0.01(\alpha-\mathrm{In})$ and $\mathrm{y}=0.5\left(\operatorname{In}_{2} \mathrm{Bi}\right)$ superconductors, respectively. The highest transition temperature was determined to be $\mathrm{T}_{\mathrm{C} 1}(10 \mathrm{Oe})=5.85 \mathrm{~K}$ and $\mathrm{T}_{\mathrm{C} 2}$ $(10 \mathrm{Oe})=4.27 \mathrm{~K}(\mathrm{y}=0.6)$ when fitted to the London equation with free fitting parameter $\mathrm{P}$. The value of $\mathrm{P}$ gives an idea about the distribution of transition temperatures and can help in naming the Meissner/flux pinning state. The SVF estimated from ZFC revealed bulk-like superconductivity for all the superconducting samples. The high and low values of flux expulsion calculated from FC revealed that $y=0.01,0.6-0.7$ are strong-linked, whereas $\mathrm{y}=0.1$ to 0.5 are relatively weak-linked intergranular superconductors. The estimated demagnetization factor $N$ $\sim 0.95(1)$ ( $y=0.1$ to 0.7 ) from field dependent magnetization measurement signals that linkage within the grains, as observed from SEM images, extends over infinity. We argue that the observed broad distribution of transition temperatures in the flux pinning state is the special feature of strong/weak-linked granular superconductors. Finally, in this report, we have demonstrated the potentiality of superconducting measurement over the SR-XRD for identifying very small volume fractions of a superconducting phase.

\section{Methods}

$\mathrm{Bi}_{\mathrm{y}} \mathrm{In}_{1-\mathrm{y}}(0.01 \leq \mathrm{y} \leq 0.7)$ superconductors were prepared by using physical solid state reaction. This tuning of the $\mathrm{Bi}$ concentration alters the crystal structure, and thereby changes its superconducting properties. Surface morphological analysis and atomic percentage calculation of all the samples were performed by field-emission scanning electron microscopy (FE-SEM) using a JEOL JSM-6500F microscope (JEOL Ltd., Tokyo, Japan). Energy dispersive spectroscopy (EDS; Inca x-sight model 7557, Oxford Instruments, Abingdon, Oxfordshire, U.K.) was utilized to estimate the atomic percentages of the constituent elements. Energy-dispersive spectroscopy (EDS) is a useful technique for estimating the atomic percentages of constituent elements in the samples.

\section{References}

1. Aditya, M. V. Superconducting State Parameters of $\mathrm{Nb}_{\mathrm{x}} \mathrm{Ta}_{\mathrm{y}} \mathrm{Mo}_{\mathrm{z}}$ Superconductors. Chinese Phys. Lett. 27, 026102 (2012).

2. Allen, P. B. \& Dynes, R. C. Transition temperature of strong-coupled superconductors reanalyzed. Phys. Rev. B 12, 905-922 (1975).

3. Hiroi, Z., Yonezawa, S., Nagao, Y. \& Yamaura, J. Extremely strong-coupling superconductivity and anomalous lattice properties in the b-pyrochlore oxide KOs2O6. Phys. Rev. B 76, 014523 (2007).

4. Takayama, T. et al. Strong Coupling Superconductivity at $8.4 \mathrm{~K}$ in an Antiperovskite Phosphide SrPt ${ }_{3}$ P. Phys. Rev. Lett. 108, 237001 (2012).

5. Cruceanu, E., Miu, L. \& Ivanciu, O. Single-crystal growth of $\operatorname{In}_{2} \mathrm{Bi}$ and $\operatorname{In}_{5} \mathrm{Bi}_{3}$ compounds. J. Cryst. Growth 28, 13-15 (1975).

6. Hutcherson, J. V., Guay, R. L. \& Herold, J. S. Superconducting In5Bi3. J. Less-Common MET. 11, 296-298 (1966).

7. Clem, J. R. \& Hao, Z. Theory for the hysteretic properties of the low-field dc magnetization in type-II superconductors. Phys. Rev. B 48, 13774-13783 (1993). 
8. Habashi, F. Alloys: Preparation, Properties, Applications. (2008).

9. Larson, A. C. \& Von Dreele, R. B. General Structure Analysis System (GSAS). Los Alamos National Laboratory Report LAUR 86, 221 (2000).

10. The XRD patterns of the $\mathrm{Bi}_{\mathrm{y}} \mathrm{In}_{1-\mathrm{y}}$ systems shows abnormally intense peaks, suggesting that most of the grains of BiIn systems are preferentially oriented with their respective hkl facets parallel to the incident X-rays.

11. Giessen, B. C. \& Grant, M. M. Trans. TMS-AIME 239 (1967)

12. Meissner, H. Superconductivity of Contacts with Interposed Barriers. Phys. Rev. 117, 672-680 (1960).

13. London, F. \& London, H. The Electromagnetic Equations of the Supraconductor. Proceedings of the Royal Society of London. Series A - Mathematical and Physical Sciences 149, 71-88 (1935).

14. Currie, P. D., Finlayson, T. R. \& Smith, T. F. The phase diagram for In-rich In-Bi alloys. J. Less-Common. MET. 62, 13-24 (1978),

15. Li, W. H., Yang, C. C., Tsao, F. C. \& Lee, K. C. Quantum size effects on the superconducting parameters of zero-dimensional Pb nanoparticles. Phys. Rev. B 68, 184507 (2003).

16. De la Peña-Seaman, O., Heid, R. \& Bohnen, K. P. Electron-phonon interaction and superconductivity in Tl-Pb-Bi alloys from first principles: Importance of spin-orbit coupling. Phys. Rev. B 86, 184507 (2012).

17. Kogan, V. G. Vortex-induced strain and magnetization in type-II superconductors. Phys. Rev. B 87, 020503 (2013).

18. Prozorov, R. Equilibrium Topology of the Intermediate State in Type-I Superconductors of Different Shapes. Phys. Rev. Lett. 98, 257001 (2007).

19. Prozorov, R., Giannetta, R. W., Polyanskii, A. A. \& Perkins, G. K. Topological hysteresis in the intermediate state of type-I superconductors. Phys. Rev. B 72, 212508 (2005).

20. Morosan, E. et al. Superconductivity in CuxTiSe2. Nat. Phys. 2, 544-550 (2006).

21. Kriener, M., Segawa, K., Ren, Z., Sasaki, S. \& Ando, Y. Bulk Superconducting Phase with a Full Energy Gap in the Doped Topological Insulator $\mathrm{CuxBi}_{2} \mathrm{Se}_{3}$. Phys. Rev. Lett. 106, 127004 (2011).

22. Bean, C. P. \& Livingston, J. D. Surface Barrier in Type-II Superconductors. Phys. Rev. Lett. 12, 14-6 (1964).

23. Konczykowski, M., Burlachkov, L. I., Yeshurun, Y. \& Holtzberg, F. Evidence for surface barriers and their effect on irreversibility and lower-critical-field measurements in Y-Ba-Cu-O crystals. Phys. Rev. B 43, 13707-10 (1991).

24. Ginzburg, V. L. The theory of superconductivity. Zh. Eksp. Teor. Fiz. 20, 1064 (1950).

25. Fetter, A. L. Superconductivity. Dekker: New York (1969).

26. Özer, M. M., Jia, Y., Zhang, Z., Thompson, J. R. \& Weitering, H. H. Tuning the Quantum Stability and Superconductivity of Ultrathin Metal Alloys. Science 316, 1594-1597 (2007).

27. Schuck, G., Kazakov, S. M., Rogacki, K., Zhigadlo, N. D. \& Karpinski, J. Crystal growth, structure, and superconducting properties of the $\beta$-pyrochlore $\mathrm{KOs}_{2} \mathrm{O}_{6}$. Phys. Rev. B 73, 144506 (2006).

28. Rogacki, K., Schuck, G., Bukowki, Z., Zhigadlo, N. D. \& Karpinski, J. Structural and superconducting properties of RbOs $\mathrm{O}_{6}$ single crystals. Phys. Rev. B 77, 134514 (2008).

29. Gandhi, A. C., Shih, P.-H. \& Wu, S. Y. Weak-coupling superconductivity in InSn nanocubes. Supercond. Sci. Technol. 25, 6 (2012).

30. Padamsee, H., Neighbor, J. E. \& Shiffman, C. A. Quasiparticle phenomenology for thermodynamics of strong-coupling superconductors. J. Low Temp. Phys. 12, 387-411 (1973).

31. Maxwell, E. \& Lutes, O. S. Threshold Field Properties of Some Superconductors. Phys. Rev. 95, 333-338 (1954).

32. McMillan, W. L. Transition Temperature of Strong-Coupled Superconductors. Phys. Rev. 167, 331-344 (1968).

33. Bardeen, J., Cooper, L. N. \& Schrieffer, J. R. Theory of Superconductivity. Phys. Rev. 108, 1175-1204 (1957).

34. Bardeen, J., Cooper, L. N. \& Schrieffer, J. R. Microscopic Theory of Superconductivity. Phys. Rev. 106, 162-164 (1957)

35. Zhang, X.-L. \& Liu, W.-M. Electron-Phonon Coupling and its implication for the superconducting topological insulators. Sci. Rep. 5, 8964 (2015).

36. Martin, I., Podolsky, D. \& Kivelson, S. A. Enhancement of superconductivity by local inhomogeneities. Phys. Rev. B 72, 060502 (2005).

37. Gao, F., Bacon, D. J., Flewitt, P. E. J. \& Lewis, T. A. The effects of electron-phonon coupling on defect production by displacement cascades in $\alpha$-iron. Modelling Simul. Mater. Sci. Eng. 6, 543 (1998).

\section{Acknowledgements}

We would like to thank the Ministry of Science and Technology (MOST) of the Republic of China for their financial support of this research through project numbers MOST-105-2112-M-259-003 and MOST-1062112-M-259-001.

\section{Author Contributions}

S.Y.W. and A.C.G. wrote, conceived, and designed the experiments. A.C.G. grew the samples and analyzed the data. All authors discussed the results, contributed to the manuscript text, commented on the manuscript, and approved its final version.

\section{Additional Information}

Supplementary information accompanies this paper at doi:10.1038/s41598-017-09831-9

Competing Interests: The authors declare that they have no competing interests.

Publisher's note: Springer Nature remains neutral with regard to jurisdictional claims in published maps and institutional affiliations.

Open Access This article is licensed under a Creative Commons Attribution 4.0 International License, which permits use, sharing, adaptation, distribution and reproduction in any medium or format, as long as you give appropriate credit to the original author(s) and the source, provide a link to the Creative Commons license, and indicate if changes were made. The images or other third party material in this article are included in the article's Creative Commons license, unless indicated otherwise in a credit line to the material. If material is not included in the article's Creative Commons license and your intended use is not permitted by statutory regulation or exceeds the permitted use, you will need to obtain permission directly from the copyright holder. To view a copy of this license, visit http://creativecommons.org/licenses/by/4.0/.

(C) The Author(s) 2017 